\title{
El Trastorno por Estrés Postraumático (PTSD) de Larga Duración como Resultado de Cirugía Genital en Menores
}

\section{Longterm Posttraumatic Stress (PTSD) Resulting From Genital Surgery in Minors}

\author{
Gregory J. Boyle ${ }^{1}$
}

\begin{abstract}
Resumen
Unos 650 millones de hombres y 120 millones de mujeres que viven en la actualidad sufrieron modificaciones genitales. Los partidarios de la cirugía genital han considerado que realizar la circuncisión y el cambio de sexo sin consentimiento en niños y niñas prácticamente no tiene secuelas psicológicas negativas. Sin embargo, la creciente evidencia empírica muestra que mutilación genital causa daño en el ámbito físico, sexual y, a veces, psicológico. Por ejemplo, de 313 hombres circuncidados cuando eran niños, el 75\% manifestaban daño psicológico, el 60\% indicaban resentimiento, el 54\% mostraban cólera, el $50 \%$ se sentían violados, el $47 \%$ se consideraban inferiores y el $43 \%$ creían que la circuncisión perjudicaba sus relaciones sexuales. Las víctimas de la circuncisión, o las sometidas a operaciones de cambio de sexo, cuando eran menores con frecuencia han descrito sus experiencias en términos de violación, tortura, mutilación y ataque sexual. Dados los efectos adversos posibles a largo plazo sobre la salud mental que causa la cirugía genital innecesaria, ha llegado el momento para que, se paren todas las formas de mutilación genital en los menores.
\end{abstract}

\begin{abstract}
An estimated 650 million males and 120 million females living today have had part of their genitals amputated during infancy or childhood. Genital surgery performed on unconsenting minors (including both circumcision and sex-reassignment surgery) has been claimed by its adherents to cause few adverse psychological effects. However, mounting evidence now reveals that such genital cutting causes harm physically, sexually, and often psychologically. For example, among 313 men circumcised as infants, $75 \%$ reported psychological harm, $60 \%$ reported resentment, $54 \%$ reported anger, $50 \%$ felt violated, $47 \%$ felt inferior to intact males, and $43 \%$ believed that circumcision impeded sexual relations. Individuals circumcised as children or subjected to sex-change
\end{abstract}

1 Based on a paper presented at the III Congreso Nacional de Psicología: "Violencia y Salud Mental", San Salvador. El Salvador, C.A., 6 y 7 Octubre 2000. e-mail: gregb@minerva.its.bond.edu.au 
operations have often described their experiences in the language of violation, torture, mutilation, and sexual assault. In view of the possible long-term adverse effects on mental health caused by unnecessary genital surgery on infants and children, it is time to cease all forms of such non-therapeutic genital cutting.

\section{Keywords: Male Circumcision, Sex-Reassignment Surgery, Sexual Reduction Surgery, Pain PTSD}

\section{Introducción}

Un acontecimiento traumático se define en DSM-IV (APA, 1994) como más allá de la experiencia humana general, tal como la agresión, el asalto sexual, o la tortura, que amenaza la integridad fisica de una persona. El trauma temprano, como la cirugía genital realizada en menores, puede tener efectos psicológicos adversos de larga duración (Levy, 1945). La circuncisión traumatiza obviamente a los menores (Eth \& Pynoos, 1985; Ramos, 2000; Terr, 1990). La circuncisión, masculina y femenina, tiene acontecimientos intensos dolorosos que implican ser fuertemente refrenado, teniendo parte de los órganos genitales amputado-cuanto más joven es el niño a la hora del trauma, mayor es la probable psicopatología subsecuente (Green, 1983). La disociación de la experiencia traumática y del dolor emocional asociado del conocimiento, es un mecanismo psicológico de defensa (Chu \& Dill, 1990; Noyes, 1977). El trauma doloroso en los menores puede causar cambios neurofisiológicos y neuroquímicos duraderos del cerebro (Ciaranello, 1983; Kolk, 1991; Anand \& Carr, 1989).

El trastorno por estrés postraumático (PTSD) puede resultar de asalto sexual, del abuso y de la violación de menores (Bownes et al., 1991; Deblinger et al., 1990; Duddle, 1991). Puede ocurrir después de la circuncisión de niños (Goldman, 1997, 1999; Menage, 1993, 1999). La comunicación presente se centra sobre todo en las consecuencias psicológicas a largo plazo de la circun- cisión masculina infantil, aunque la circuncisión femenina (Abd el Salam, 1999; Elchalal et al., 1999; Toubia, 1999) y la cirugía amputada del cambio de sexo realizada en menores que no han dado su consentimiento (AAP, 2000) también representan un abuso sexual serio, dejando a menudo a las víctimas con cicatrices emocionales de por vida.

\section{Efectos de la Circuncisión}

Los hombres circuncidados que han reconocido la pérdida de una pieza altamente erógena e irreemplazable de su pene han señalado sufrimiento emocional de larga duración, la pena, la ansiedad, y la depresión, y un sentido de la vulnerabilidad personal. La evitación o la preocupación obsesiva con tal pérdida, junto con cólera puede ser dificil de reconciliar para algunos hombres dependiendo de su personalidad (Bigelow, 1995; Kolk, 1989; Maguire, 1998).

La circuncisión infantil puede afectar negativamente su bienestar sexual futuro. En una encuesta de 313 hombres circuncidados (Hammond, 1997), el reporte de daños de la circuncisión incluía lo siguiente: daño sexual (84\%), daño emocional (83\%), daño fisico $(82 \%)$, daño psicológico $(75 \%)$, autoestima baja (74\%), problemas con la intimidad (45\%), problemas de adicción/dependencia (26\%). Los problemas fisicos resultantes incluían: la insensibilidad del glande del pene (55\%), necesidad de exceso del estímulo para permitir la eyaculación (38\%), 
cicatriz prominente (29\%), y escasa piel residual del pene (27\%). También se detectaron problemas psicológicos: sentimiento de insatisfacción con el estatus circuncidado $(69 \%)$, sentirse mutilado $(62 \%)$, sentirse incompleto $(61 \%)$, resentimiento $(60 \%)$, sentirse anormal/antinatural $(60 \%)$, sentir que sus derechos humanos habían sido infringidos $(60 \%)$, sentirse enojado (54\%), frustrado $(53 \%)$, violado (50\%), inferior a los hombres intactos $(47 \%)$, impedido sexualmente $(43 \%)$, y sentirse traicionado por los padres (34\%).

Con una muestra creciente de 546 hombres circuncidados, Hammond (1999) encontró que $61 \%$ mostraron déficit sensorial progresivo en el remanente interno del prepucio y en el glande del pene, junto con una disfunción eréctil, dificultad en las eyaculaciónes, y/o sin orgasmo. Inversamente, la disfunción sexual que resultaba de la señal de la alteración emocional de ser circuncidado también fue encontrada. De hecho, $41 \%$ señaló que su sufrimiento fisico y emocional impidió intimidad emocional con sus parejas. Hammond (1999) señala que la alteración emocional y pensamientos recurrentes sobre su circuncisión incluye sensaciones de mutilación (60\%), de autoestima baja y de inferioridad (50\%), de dysmorphia genital (55\%), enojo (52\%), resentimiento y depresión (59\%), de violación $(46 \%)$, y de traición paterna (30\%).

Los hombres circuncidados tuvieron que recurrir a menudo a relaciones sexuales prolongadas para estimular las terminaciónes nerviosas residuales del pene suficientemente para accionar la eyaculación. Señalaron que la sequedad artificial de su pene circuncidado a menudo hacía el coito doloroso. O'Hara y O'Hara (1999) encontraron que las parejas del sexo femenino señalaron placer sexual perceptiblemente mayor de la copulación con los hombres intactos con respecto a hombres circuncidados (aunque este déficit se ha atenuado cuando hombres circuncidados han restablecido un prepucio su- cedáneo con el proceso de la extensión de la piel-ver Bigelow, 1995).

Money y Davison (1983) examinaron las consecuencias erotogénicas de la circuncisión de hombres adultos (menos debilitante que la circuncisión infantil) documentaron una pérdida de receptores proprioceptivos del estiramiento (del prepucio y el frenillo), respuesta sexual disminuida, dolor peneal aumentado y los cambios resultados en la técnica de despertamiento o de masturbación. La exposición al dolor intenso libera hormonas considerables de estrés durante la circuncisión neonatal, que puede tener consecuencias psicobiológicas adversas que potencialmente alteran desfavorablemente el desarrollo del cerebro, la función sexual, y el comportamiento (Prescott, 1989).

Un estudio antes-y-después de 12 muchachos turcos que habrían sido circuncidados, Cansever (1965) señaló que «la circuncisión es percibida por el niño como un ataque agresivo sobre su cuerpo, que daña, mutila y en algunos casos, los destruye totalmente.» La circuncisión ritual dio lugar a agresividad creciente, al debilitamiento del ego, a la retirada, a la adaptación reducida, y a pesadillas consecuentes con un diagnóstico de PTSD. McFadyen (1998) señaló el trauma psicológico experimentado por su hijo después de la circuncisión, consistente con los informes tempranos de Anna Freud (1952).

Taddio et al. (1997) documentaron sensibilidad aumentada para el dolor en bebés seis meses después de la circuncisión, sugestiva de PTSD. Rhinehart (1999) señaló PTSD en hombres de mediana edad sometidos circuncisión infantil. Anand y Scalzo (2000) sugirieron que el trauma temprano conduce a la sensibilidad creciente del dolor, a los desórdenes del estrés, a ADHD (Trastorno Hiperactivo de la Atención Deficitaria), y as comportamientos auto destructivos. Hay también evidencia que 
el trauma temprano (tal como circuncisión neonatal) contribuye a un comportamiento posterior agresivo, violento y suicida (Jacobson et al., 1987; Parkes, 1998; Bradley et al., 1998; Jacobson \& Bygdeman, 1998).

Sigmund Freud (1920) afirmó que la circuncisión era un substituto para la castración (cf. Ozturk, 1973). Más recientemente, Immerman y Mackey (1998) describieron la circuncisión como "castración neurológica de calidad inferior.» Discutieron que la queratinización resultante del glande del pene y la atrofia neurológica del circuito del cerebro (debido a la pérdida de entrada de información sensorial al centro del placer del cerebro) serían quizás un mecanismo sociológico para producir un varón que es menos excitable sexualmente y más adecuado a los condicionamientos sociales. Los hombres circuncidados recordaron tal ansiedad de la castración y los miedos asociados a la circuncisión hecha en forma obligada en su niñez. El entumecimiento emocional, la evitación del asunto de la circuncisión, y la cólera son consecuencias psicológicas a largo plazo potenciales del trauma de la circuncisión (Bigelow, 1995; Goldman, 1997, 1999; Hammond, 1997, 1999; Rhinehart, 1999).

La circuncisión en niños implica un desequilibrio de fuerza entre el autor y la víctima. Contiene elementos agresivos y libidinales, y la integridad sexual y corporal del niño es violada por la amputación de una parte de sus órganos genitales. Los adultos que eran circuncidados como los niños (siempre sin su consentimiento) han descrito sus experiencias en el lenguaje de la violación, de la tortura, de la mutilación, y del asalto sexual (Bigelow, 1995; Hammond, 1999). En un estudio, un cuestionario clínico validado (PSTD-I) con una escala de respuesta siete puntos (Watson et al., 1991) fue administrado por Menage (1999) a ocho hombres que habían sido circuncidados cuando eran niños. Seis de los ocho hombres satisficieron los criterios de un diagnóstico de PTSD.

Ramos (2000) condujo un estudio sobre los efectos psicológicos asociados a procedimientos médicos y rituales de la circuncisión en las Filipinas. Unos 1577 muchachos en edades de 11 a 16 años (1072 muchachos fueron circuncidados bajo procedimientos médicos; 505 fueron sometidos a circuncisión ritual) fueron investigados para ver si el trauma percibido del corte genital condujo al desarrollo de PTSD. Usando el cuestionario de PTSD-I (Watson et al., 1991), Ramos encontró que casi $70 \%$ de los muchachos sometidos a la circuncisión ritual, y $51 \%$ de los muchachos sometidos a circuncisión médica satisficieron los criterios para un diagnóstico de PTSD. Este estudio sugiere que hay necesidad de informar a la comunidad filipina sobre el daño psicológico causado por la circuncisión, tanto ritual como médica. En términos de implicaciones médico-legales, un muchacho traumatizado psicológicamente por una circuncisión hecha podría tener argumentos para buscar la compensación legal (véase Boyle et al., 2000).

\section{Efectos de Cambio de Sexo}

En vista de la cirugía de cambio de sexo impuesta a millares de niños por todo el mundo nacidos con los órganos genitales ambiguos, o en niños masculinos que han sufrido la necrosis iatrogénica del pene por circuncisiones mal hechas que eran en primer lugar totalmente innecesarias, la evidencia ahora indica que la gran mayoría de las víctimas adultas rechazan lo que se les ha hecho cuando eran niños (Bradley et al., 1998; Colapinto, 1997; Diamond, 1997; Kipnis \& Diamond, 1998; Wilson \& Reiner, 1999). Muchos de estos individuos parecen sufrir PTSD a largo plazo (McMullen, 2000). El corte genital impuesto en menores viola claramente los derechos humanos 
de los niños, reduce inevitablemente la capacidad para el placer y el orgasmo sexuales, quita capacidad reproductiva (e.g., en casos de la castración), y causa a menudo el estrés psicológico de por vida, puesto que la víctima es atrapada en el cuerpo «del sexo opuesto.»

Claramente, la cirugía de cambio de sexo debe ser pospuesta hasta que el individuo implicado ha alcanzado la edad adulta, momento en el cual habría emergido la identidad del género decisivo, y él o ella pueda tener una opción al estar completemente informado. La posición de la Sociedad del Intersexo de Norteamérica es que cada uno tiene derecho a todos sus órganos genitales y que solamente tienen derecho a decidir que parte de su cuerpo no desean (Milos, 2000). El hospital de la Universidad Johns Hopkins que ha estado a la vanguardia promoviendo la cirugía de cambio de sexo en menores que no han dado su consentimiento, ha llamado ahora a una moratoria de tal cirugía involuntaria, poca ética, a la luz de la evidencia de daño a largo plazo y PTSD que ahora está emergiendo (e.g., Colapinto, 1997; McMullin, 2000). La Corte Constituciónal de Colombia (véase Greenberg \& Chase, 2000) ha declarado recientemente que la cirugía forzosa es ilegal en menores, $y$ ha limitado significativamente la capacidad de los médicos y los padres para alterar o amputar quirúrgicamente los órganos sexuales de niños con genitales ambiguos y víctimas de circuncisiones mal hechas.

\section{Conclusiones}

En vista que la pruebas crecientes de las cirugías genitales realizadas en menores que no han dado su consentimiento, incluyendo la circuncisión masculina y femenina, así como la cirugía del cambio de sexo, dan lugar inevitablemente a daño fisico, sexual, y a menudo psicológico de por vida, es hora de llamar a un alto mundial a todas las formas de corte genital. La cirugía genital involuntaria, irreversible, impulsada en los infantes y los niños tiene a menudo efectos dañinos a largo plazo en la salud mental.

\section{Referencias}

ABd el Salam, S. (1999). A comprehensive approach for communication about female genital mutilation in Egypt. In G. C. Denniston et al. (Eds), Male and female circumcision: Medical, legal, and ethical considerations in pediatric practice. New York: Plenum. Pp. 317-330.

American academy of Pediatrics (2000). Evaluation of the newborn with developmental anomalies of the external genitalia (RE9958). Pediatrics, 106, 138142.

ANAND, K. J, \& CARR, D. (1989). The neuroanatomy, neurophysiology, and neurochemistry of pain, stress, and analgesia in newborns and children. Pediatric Clinic of North America, 36, 795-822.

Anand, K. J., \& Scalzo, F. M. (2000). Can adverse neonatal experiences alter brain development and subsequent behavior? Biology of the Neonate, 77, 69-82.

Bensley, G. A. (1999). Physical, sexual, and psychological impact of male infant circumcision: An exploratory survey. Unpublished Honours Thesis, Bond University.

BIGELOW, J. (1992). The joy of uncircumcising: Exploring circumcision: History, myths, psychology, restoration, sexual pleasure and human rights. Aptos, CA: Hourglass.

Bownes, I. T., O'Gorman, E. C., \& SAYers, A. (1991). Assault characteristics and post-traumatic stress disorder in rape victims._Acta Psychiatrica Scandinavica, 83, 27-30. 
Boyle, G. J., Svoboda, J. S., Price, C. P., \& Turner, J. N. (2000). Circumcision of healthy boys: Criminal assault? Journal of Law and Medicine, 7, 301-310. See http:/ /www.cirp.org/library/legal/boyle1

Bradley, S. J., Oliver, G. D., Chernick, A. B., \& ZUCKER, K. J. (1998). Experiment of nurture: Ablatio penis at 2 months, sex reassignment at 7 months, and a psychosexual follow-up in young adulthood. Pediatrics, 102, e9.

Cansever, G. (1965)._Psychological effects of circumcision. British Journal of Medical Psychology, 38, 328.

Chu, J., \& Dill, D. (1990). Dissociative symptoms in relation to childhood physical and sexual abuse. American Journal of Psychiatry, 147, 887-892.

Ciaranello, R. (1983). Neurochemical aspects of stress. In N. Garmezy \& M. Rutter (Eds), Stress, coping, and development. New York: McGraw-Hill.

Colapinto, J. (1997). The true story of John/ Joan. Rolling Stone, December, 54-97.

Deblinger, E., McLeer, S. V., \& Henry, D. (1990). Cognitive behavioural treatment for sexually abused children suffering post-traumatic stress. Journal of the AmericanAcademy of Child and Adolescent Psychiatry, 29, 747-752.

Diagnostic and Statistical Manual of MenTAL DisORDERS, FOURTH EDITION (DSM-IV). (1994). Washington, DC: American Psychiatric Association.

DiAMOND, M. (1997). Sex reassignment at birth: A long term review and clinical implications. Archives of Pediatric and Adolescent Medicine, 151, 298-304.

Duddle, M. (1991). Emotional sequelae of sexual assault. Journal of the Royal Society of Medicine, 84, 26-28.
Elchalal, U., Ben-Ami, B., \& BRZeZINSKI, A. (1999). Female circumcision: The peril remains. British Journal of Urology International, 83, Supplement 1, 103-108.

Eth, S., \& Pynoos, R. (1985). Developmental perspective on psychic trauma in childhood. In C. Figley (Ed), Trauma and its wake. New York: Brunner/Mazel.

FREUD, A. (1952). The role of bodily illness in the mental life of children. Psychoanalytic Study of the Child, 7, 69-81.

FREUD, S. (1966). Introductory lectures on psychoanalysis (1920 reprint). New York: Norton, p. 165.

Goldman, R. (1997). Circumcision: The hidden trauma. Boston: Vanguard.

Goldman, R. (1999). The psychological impact of circumcision. British Journal of Urology International, 83, Supplement 1, 93-102._See http://www.cirp.org/ library/psych/goldman1

Greenberg, J. A., \& Chase, C. (2000). Colombia High Court limits surgery on intersexed infants. See http://www.isna.org/ colombia/background.html

HAMmOND, T. (1997). Long-term consequences of neonatal circumcision: A preliminary poll of circumcised males. In G. C. Denniston \& M. F. Milos (Eds), Sexual mutilations: A human tragedy. New York: Plenum.

HAMmOND, T. (1999). A preliminary poll of men circumcised in infancy or childhood. British Journal of Urology International, 83, Supplement 1, 85-92.

Immerman, R. S., \& Mackey, W. C. (1998). A proposed relationship between circumcision and neural reorganization. Journal of Genetic Psychology, 159, 367-378. 
Jacobson, B., \& Bygdeman, M. (1998). Obstetric care and proneness of offspring to suicide. British Medical Journal, 317, 1346-1349.

Jacobson, B., EkLund, G., Hamburger, L., LinNarsson, D., Sedvall, G., \& Valverius, M. (1987). Perinatal origin of adult selfdestructive behavior. Acta Psychiatrica Scandinavica, 76, 364-371.

KIPNIS, K., \& DiAmond, M. (1998). Pediatric ethics and the surgical assignment of sex. Journal of Clinical Ethics, 9, 398-410.

KOLK, VAN DER B. (1989). The compulsion to repeat the trauma: Re-enactment, revictimization, and masochism. Psychological Clinics of North America, 12, 389-411.

Levy, D. M. (1945). Psychic trauma of operations in children. American Journal of Diseases of Children, 69, 7-25.

Maguire, P. (1998). Coping with loss: Surgery and loss of body parts. British Medical Journal, 316, 1086-1088.

McFADYEN, A. (1998). Children have feelings too. British Medical Journal, 316, 1616.

McMullen, J. (2000). Breaking the silence. 60 Minutes national television program aired in Australia, June 25.

Menage, J. (1999). Post-traumatic stress disorder after genital medical procedures. In G. C. Denniston, F. M. Hodges, \& M. F. Milos (Eds), Male and female circumcision: Medical, legal, and ethical considerations in pediatric practice. New York: Kluwer Academic/Plenum.

Menage, J. (1993). Post-traumatic stress disorder in women who have undergone obstetric and/or gynaecological procedures. Journal of Reproductive and Infant Psychology, 11, 221-228.
Milos, M. F. (2000). Personal communication, June 6. NOCIRC at http://www.nocirc.org

MoneY, J., \& Davison, J. (1983). Adult penile circumcision: Erotosexual and cosmetic sequelae. Journal of Sex Research, 19, 289292.

NOYEs, R. (1977). Depersonalization in response to life threatening danger. Comprehensive Psychiatry, 18, 375-384.

O'Hara, K., \& O'Hara, J. (1999). The effect of male circumcision on the sexual enjoyment of the female partner. British Journal of Urology International, 83, Supplement 1, 79-84.

Ozturk, O. (1973). Ritual circumcision and castration anxiety. Psychiatry, 36, 55.

PARKes, C. M. (1998). Coping with loss: Facing loss._British Medical Journal, 316, 1521-1524.

Prescott, J. W. (1989). Genital pain vs. genital pleasure: Why the one and not the other? Truth Seeker, 1, 14-21.

Ramos, S. (2000). Ritual and medical circumcision among Filipino boys: Evidence of post-traumatic stress disorder. Unpublished Honours Thesis, Bond University.

RHINEHART, J. (1999). Neonatal circumcision reconsidered. Transactional Analysis Journal, 29, 215-221.

TERR, L. (1990). Too scared to cry. New York: Harper \& Row.

Toubia, N. F. (1999). s. Evolutionary cultural ethics and the circumcision of children In G. C. Denniston et al. (Eds), Male and female circumcision: Medical, legal, and ethical considerations in pediatric practice. New York: Plenum. Pp. 1-7. 
Watson, C. G., Juba, M. P., Manifold, V., Kucala, T., \& Anderson, P. E. D. (1991). The PTSD interview: Rationale, description, reliability, and concurrent validity of a DSM-III-based technique. Journal of Clinical Psychology, 47, 179-188.
Wilson, B. E., \& Reiner, W. G. (1999). Management of intersex: A shifting paradigm. In A. Dreger (Ed), Intersex in the age of ethics. University Press, University of Maryland, USA. 